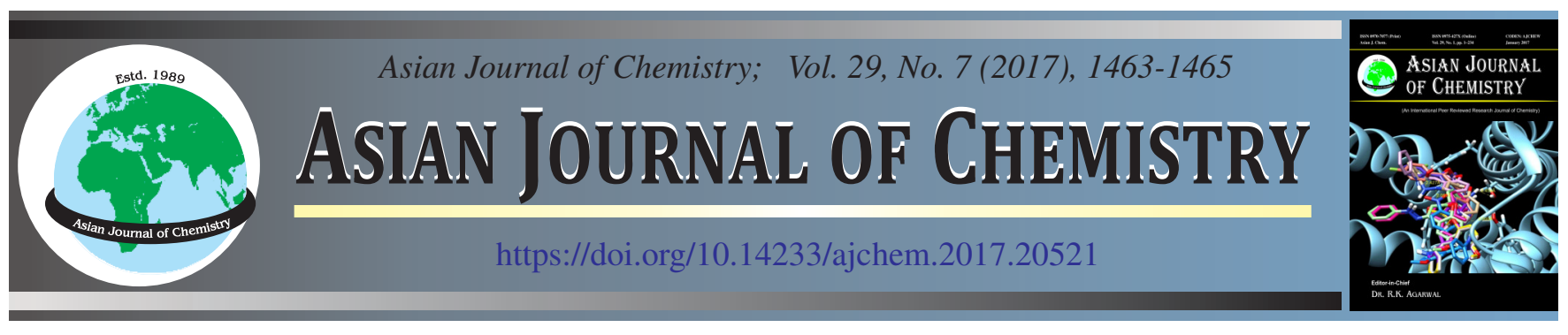

\title{
Synthesis and Characterization of Some Homonuclear Bimetallic Complexes with Macrocyclic Ligands: A Photoelectron Spectroscopic Study
}

\author{
Abhishek Kumar*, Shishir Malviya, Rajesh Kumar Kushwaha and Shekhar Srivastava
}

Department of Chemistry, University of Allahabad, Allahabad-211 002, India

*Corresponding author: E-mail: abhishekrajpoot02@gmail.com

Received: 17 January 2017;

Accepted: 7 March 2017;

Published online: 13 May 2017;

AJC-18374

We have synthesized bimetallic macrocyclic complexes through chemical condensation method by using pyridine-2,6-dicarboxaldehyde with 1,2-bis(2-aminoethoxy) ethane where $\mathrm{L}$ is the macrocyclic ligand. The synthesized complexes were characterized by elemental analysis, IR, XPS data, NMR and UV-visible spectroscopy.

Keywords: Binuclear complexes, Heavy metals, X-ray photoelectron spectroscopy, Macrocyclic complexes.

ᄂ

\section{INTRODUCTION}

Macrocyclic ligands have been a subject of extensively investigated in coordination chemistry research due to ability to form complexes with different metal ions [1,2]. Several examples of such type of ligands as a crown ethers, porphyrin and saturated or unsaturated macrocyclic polyamines are well known and their chemical properties and function as metal complexes have been systematically heightened by the facile and diversified chemical modification of their macrocyclic framework [3]. A large number of macrocycles [4-6] and their complexes with transition metal ion have been synthesized and characterized. Therefore the design and study of macrocyclic complexes with desirable properties is still a notable achievement. The direct synthesis or template synthesis is the heart of macrocyclic chemistry and have been widely used for synthesis of macrocyclic complexes where transition metal ions are widely used as templating metal agents [7]. The application of macrocyclic complexes are associated with their ability to complex metal ions. The main target to synthesized macrocyclic ligands which are able to discriminate between metal ions. The ability to control metal ion selectivity is clearly great interest in many areas and this selection is influenced by the nature, arrangement of donor atom and the ring size. Macrocyclic complexes are signicant as they can be used as catalysts in many organic oxidation reactions [8] and they have been found to act as possible models for biochemically important proteins and enzymes [9]. Recently several macrocyclic complexes are reported with different metal ions [10-16].
On the basis of these facts, we have synthesized macrocyclic complexes with $\mathrm{Cu}(\mathrm{II}), \mathrm{Pb}(\mathrm{II}), \mathrm{Zn}(\mathrm{II}), \mathrm{Ni}(\mathrm{II})$ and Co(II) with macrocyclic ligand (L) derived from pyridine-2,6dicarboxaldehyde with 1,2-bis(2-aminoethoxy)ethane through chemical condensation method. All the synthesized complexes $\left[\mathrm{M}_{2} \cdot \mathrm{L}\right]$ were characterized by elemental analysis, IR, XPS data, NMR and UV-visible spectroscopy. IR spectra in $\mathrm{KBr}$ were recorded on a Perkin-Elmer 457 IR spectrophotometer. Elemental analysis was carried out in a Coleman automatic carbon, hydrogen and nitrogen analyser. The X-ray photo-electron spectra i.e. XPS were recorded on a VG scientific ESCA-3MK II electron spectrometer.

\section{EXPERIMENTAL}

All the chemical reagents were of analytical grade and purchased commercially and used without further purification.

Preparation of ligand: The ligand (L) was prepared by chemical condensation method with the reaction between 2,6pyridine-dicarboxaldehyde with 1,2-bis(2-aminoethoxy)ethane (1:1) molar ratio in dimethyl formamide (DMF) solution. We take $40 \mathrm{~mL}$ DMF solution, pyridine-2,6-dicarboxaldehyde and metal salt $\left(\mathrm{MX}_{2}\right)$ in a round bottle flask after complete dissolution of both the reagents a solution of 1,2-bis (2-aminoethoxy)ethane was added drop-wise. After $1 \mathrm{~h}$ stirring at $55^{\circ} \mathrm{C}$ pale yellow colour complexes were obtained with yield $70 \%$. After reduction with $\mathrm{NaBH}_{4}$ in methanol solution and stirring continuously $3 \mathrm{~h}$. Then we used demetallation technique using $\mathrm{H}_{4}$ EDTA chelator with chloroform give the metal free reduced macrocyclic ligand (L) as a pale yellow oil. Yield $35 \% . \mathrm{N} 1 \mathrm{~s}$ 
binding energies $399.6 \mathrm{eV}$ compared to coordinated $\mathrm{N}$ 1s binding energies $401.4 \mathrm{eV}$ showed that all $\mathrm{N}$ of ligand $(\mathrm{L})$ are uncoordinated (Table-1). ESI-MS: $m / z$ 503.4 (M $\left.{ }^{+}\right) .{ }^{1} \mathrm{H}$ NMR $\left(\mathrm{CDCl}_{3}, 400 \mathrm{MHz}, \delta \mathrm{ppm}\right): 7.52-7.57$ (a, triplet), 7.17 (b, doublet), 3.80 (c, doublet), 3.62-3.78 (e + f, triplet), 2.87 (d, triplet), 2.29 (g, singlet).

TABLE-1

$\mathrm{N}$ 1s BINDING ENERGIES (eV) IN $\left[\mathrm{M}_{2} \cdot \mathrm{L}\right] \cdot \mathrm{X}_{4}$, WHERE $\mathrm{M}=\mathrm{Pb}(\mathrm{II})$, $\mathrm{Zn}(\mathrm{II}), \mathrm{Ni}(\mathrm{II}), \mathrm{Cu}$ (II) OR $\mathrm{Co}(\mathrm{II}), \mathrm{X}=\mathrm{SCN}^{-}, \mathrm{Cl}^{-} \mathrm{OR} \mathrm{Br}$

\begin{tabular}{ccc}
\hline \multirow{2}{*}{ Compound } & \multicolumn{2}{c}{$\mathrm{N} 1 \mathrm{~s}$} \\
\cline { 2 - 3 } & Uncoordinated & Coordinated \\
\hline$\left[\mathrm{Pb}_{2} \cdot \mathrm{L}\right] \cdot \mathrm{X}_{4}$ & $399.4(6)$ & $403.6(4)$ \\
{$\left[\mathrm{Zn}_{2} \cdot \mathrm{L}\right] \cdot \mathrm{X}_{4}$} & $399.8(4)$ & $403.4(8)$ \\
{$\left[\mathrm{Ni}_{2} \cdot \mathrm{L}\right] \cdot \mathrm{X}_{4}$} & $399.4(6)$ & $403.6(4)$ \\
{$\left[\mathrm{Cu}_{2} \cdot \mathrm{L}\right] \cdot \mathrm{X}_{4}$} & $399.6(4)$ & $403.6(4)$ \\
{$\left[\mathrm{Co}_{2} \cdot \mathrm{L}\right] \cdot \mathrm{X}_{4}$} & $399.4(8)$ & $403.4(5)$ \\
\hline
\end{tabular}

Preparation of $\left[\mathrm{M}_{2} \cdot \mathrm{L}\right] \mathrm{X}_{4}$ complexes where $\mathrm{M}=\mathrm{Pb}(\mathrm{II})$, $\mathrm{Zn}(\mathrm{II}), \mathrm{Ni}(\mathrm{II}) \mathrm{Cu}(\mathrm{II})$ and $\mathrm{Co}(\mathrm{II}),\left(\mathrm{X}=\mathrm{SCN}^{-}, \mathrm{Cl}^{-}\right.$and $\left.\mathrm{Br}^{-}\right)$: Metal salts of $\mathrm{MX}_{2}$ and 2,6-pyridine-dicarboxaldehyde (1:1) molar ratio in round bottle flask containing $40 \mathrm{~mL}$ dimethyl formamide solution. After complete dissolution of both the reagents 1,2-bis(2-aminoethoxy) ethane solution was added drop-wise. After $1 \mathrm{~h}$ stirring at $55{ }^{\circ} \mathrm{C}$ colour complexes were obtained with good yield. All the macrocyclic complexes are stable at room temperature.

\section{RESULTS AND DISCUSSION}

The binding energies $(\mathrm{eV})$ of $\mathrm{M} 3 \mathrm{p}_{1 / 2,3 / 2}$ in $\left[\mathrm{M}_{2} \cdot \mathrm{L}\right] \cdot \mathrm{X}_{4}$ [where $\mathrm{M}=\mathrm{Pb}(\mathrm{II}), \mathrm{Zn}(\mathrm{II}), \mathrm{Ni}(\mathrm{II}), \mathrm{Cu}(\mathrm{II})$ and $\mathrm{Co}(\mathrm{II}), \mathrm{X}=\mathrm{SCN}^{-}, \mathrm{Cl}^{-}$ and $\left.\mathrm{Br}^{-}\right]$and $\mathrm{N} 1 \mathrm{~s}$ photoelectron peaks are listed in Tables 1-3. It was observed that the binding energies of $\mathrm{M} 3 \mathrm{p}_{1 / 2}, 3 / 2$ in ligand (L) was lower than their prepared molecular adducts i.e. $\left[\mathrm{M}_{2} \cdot \mathrm{L}\right] \mathrm{X}_{4}$. These observation suggested that electron density on the M(II) metal ion has increased due to coordination of ligands with M(II) metal ion [16] (Fig. 1). Moreover, N1s photoelectron peaks with intensity ratio $6: 6$ in $\left[\mathrm{M}_{2} \cdot \mathrm{L}\right] \cdot \mathrm{X}_{4}$ at $399.4(\mathrm{eV})$ suggested that all the six nitrogen are coordinated and at $403.6(\mathrm{eV})$ in ligand suggested that all six nitrogen are nitrogen are uncoordinated [16] (Fig. 1, Tables 1-3). These

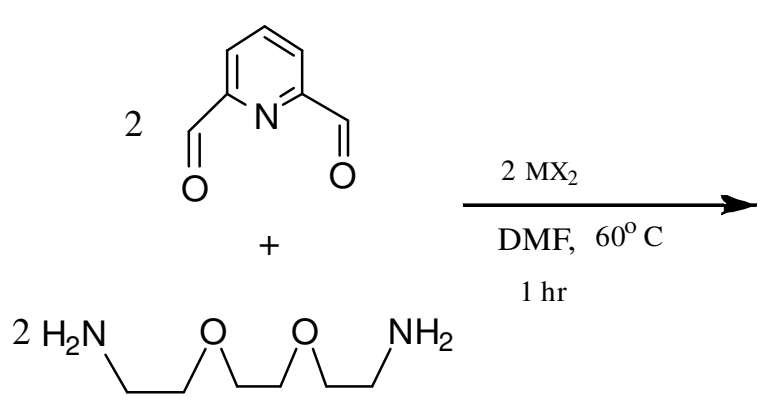

Where $\mathrm{M}=\mathrm{Pb}$ (II), $\mathrm{Zn}(\mathrm{II}), \mathrm{Ni}(\mathrm{II})$, $\mathrm{Cu}(\mathrm{II})$ and $\mathrm{Co}$ (II)

$\mathrm{X}=\mathrm{SCN}^{-}, \mathrm{Cl}^{-}, \mathrm{Br}^{-}$

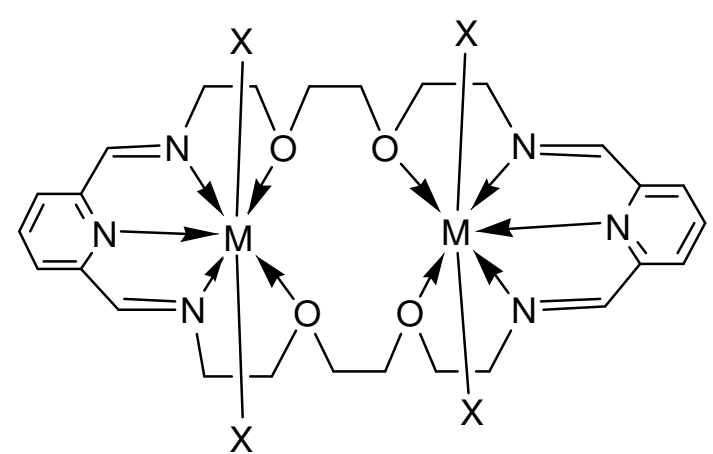

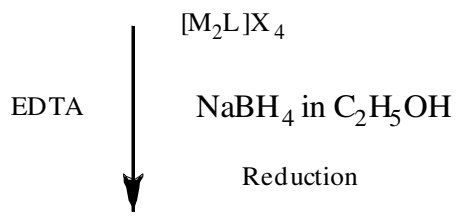

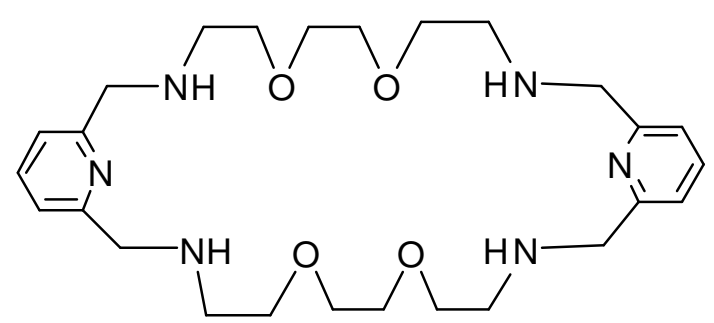

Reduce lig and (L)

Fig. 1. Scheme for the preparation of metal complexes $\left[M_{2} L\right] \cdot X_{4}$

TABLE-2

ANALYTICAL DATA OF $\left[\mathrm{M}_{2} \cdot \mathrm{L}\right] \mathrm{X}_{4}$ WHERE $\mathrm{M}=\mathrm{Pb}(\mathrm{II}), \mathrm{Zn}(\mathrm{II}), \mathrm{Ni}(\mathrm{II}), \mathrm{Cu}$ (II) OR $\mathrm{Co}(\mathrm{II}), \mathrm{X}=\mathrm{SCN}^{-}, \mathrm{Cl}^{-} \mathrm{OR} \mathrm{Br}^{-}$

\begin{tabular}{|c|c|c|c|c|c|c|}
\hline \multirow{2}{*}{ Compound } & \multicolumn{5}{|c|}{ Elemental analysis (\%): Found (Calculated) } & \multirow{2}{*}{$\begin{array}{l}\text { Molar conductance } \\
\left(\mathrm{ohm}^{-1} \mathrm{~cm}^{2} \mathrm{~mol}^{-1}\right)\end{array}$} \\
\hline & $\mathrm{C}$ & $\mathrm{H}$ & $\mathrm{N}$ & $\mathrm{O}$ & $\mathrm{M}$ & \\
\hline$\left[\mathrm{Pb}_{2} \cdot \mathrm{L}\right] \cdot \mathrm{X}_{4}$ & $34.2(34.4)$ & $3.6(3.7)$ & $9.0(9.2)$ & $7.2(7.0)$ & $45.5(45.6)$ & 24 \\
\hline$\left[\mathrm{Zn}_{2} \cdot \mathrm{L}\right] \cdot \mathrm{X}_{4}$ & $49.8(49.9)$ & $5.4(5.5)$ & $13.3(13.4)$ & $10.0(10.2)$ & $20.8(20.9)$ & 22 \\
\hline$\left[\mathrm{Ni}_{2} \cdot \mathrm{L}\right] \cdot \mathrm{X}_{4}$ & $50.4(51.0)$ & $5.4(5.6)$ & $13.4(13.7)$ & $10.3(10.5)$ & $19.6(19.8)$ & 26 \\
\hline$\left[\mathrm{Cu}_{2} \cdot \mathrm{L}\right] \cdot \mathrm{X}_{4}$ & $50.6(50.2)$ & $5.7(5.5)$ & $13.2(13.6)$ & $10.4(10.2)$ & $20.2(20.4)$ & 30 \\
\hline$\left[\mathrm{Co}_{2} \cdot \mathrm{L}\right] \cdot \mathrm{X}_{4}$ & $50.6(51.0)$ & $5.4(5.6)$ & $13.2(13.7)$ & $10.3(10.5)$ & $19.4(19.2)$ & 28 \\
\hline
\end{tabular}




\begin{tabular}{|c|c|c|}
\hline \multicolumn{3}{|c|}{$\begin{array}{c}\text { TABLE-3 } \\
\text { M 3 } \mathrm{p}_{1 / 2,3 / 2} \text { BINDING ENERGIES (eV) in }\left[\mathrm{M}_{2} \cdot \mathrm{L}^{2} \cdot \mathrm{X}_{4} \text { WHERE } \mathrm{M}=\right. \\
\mathrm{Pb}(\mathrm{II}), \mathrm{Zn}(\mathrm{II}), \mathrm{Ni}(\mathrm{II}), \mathrm{Cu}(\mathrm{II}) \text { or } \mathrm{Co}(\mathrm{II}), \mathrm{X}=\mathrm{SCN}^{-}, \mathrm{Cl}^{-} \text {or } \mathrm{Br}^{-}\end{array}$} \\
\hline \multirow{2}{*}{ Compound } & \multicolumn{2}{|c|}{$\mathrm{Pb} \mathrm{3} \mathrm{p}_{1 / 2,3,2}$} \\
\hline & $\mathrm{Pb} \mathrm{3p_{1/2 }}$ & $\mathrm{Pb} \mathrm{3} \mathrm{p}_{3 / 2}$ \\
\hline$\left[\mathrm{Pb}_{2} \cdot \mathrm{L}\right] \cdot \mathrm{X}_{4}$ & 568.4 & 542.4 \\
\hline$\left[\mathrm{Zn}_{2} \cdot \mathrm{L}\right] \cdot \mathrm{X}_{4}$ & 1056.6 & 1032.6 \\
\hline$\left[\mathrm{Ni}_{2} \cdot \mathrm{L}\right] \cdot \mathrm{X}_{4}$ & 884.4 & 864.6 \\
\hline$\left[\mathrm{Cu}_{2} \cdot \mathrm{L}\right] \cdot \mathrm{X}_{4}$ & 962.4 & 931.6 \\
\hline$\left[\mathrm{Co}_{2} \cdot \mathrm{L}\right] \cdot \mathrm{X}_{4}$ & 798.6 & 790.4 \\
\hline
\end{tabular}

metal complexes show strong IR band near 1660-1625 and $1600-1560$ are suggested to $v(\mathrm{C}=\mathrm{N})$ and highest energy pyridine ring vibration respectively [15].

\section{Conclusion}

All the complexes were synthesized are of good yield. The effect of ligand $(\mathrm{N})$ coordination with metal by the help $\mathrm{M} 3 \mathrm{p}_{1 / 2,3 / 2}$ and N1s binding energies also showed. On the basis of elemental analysis, IR and XPS data, the structural geometry of above complexes are determined as octahedral geometry.

\section{ACKNOWLEDGEMENTS}

One of the authors, Abhishek Kumar is thankful to Department of Chemistry, University of Allahabad, Allahabad, India and University Grants Commission (UGC) for providing the financial assistance.

\section{REFERENCES}

1. R.M. Izatt, J.S. Bradshaw, S.A. Nielsen, J.D. Lamb, J.J. Christensen and D. Sen, Chem. Rev., 85, 271 (1985);

https://doi.org/10.1021/cr00068a003
2. K.B. Mertes, J.M. Lehnin and G. Wilkinsion, Comprehensive Coordination Chemistry, Pergamon, Oxford, p. 915 (1987).

3. G.W. Gokel, W.M. Leevy and M.E. Weber, Chem. Rev., 104, 2723 (2004); https://doi.org/10.1021/cr020080k.

4. D.H. Busch, Acc. Chem. Res., 11, 392 (1978); https://doi.org/10.1021/ar50130a005.

5. J.S. Trommel and L.G. Marzilli, Inorg. Chem., 40, 4374 (2001); https://doi.org/10.1021/ic010232e.

6. X. Lu, Z. Geng, Y. Wang, B. Lv and J. Kang, Synth. React. Inorg. Met.-Org. Chem., 32, 843 (2002); https://doi.org/10.1081/SIM-120005606.

7. M.S. Niasari and F. Davar, Inorg. Chem. Commun., 9, 175 (2006); https://doi.org/10.1016/j.inoche.2005.10.028.

8. C.M. Che and W.K. Cheng, J. Chem. Soc. Commun., 1443 (1986); https://doi.org/10.1039/c39860001443.

9. L. Casella, M. Gullotti, L. De Gioia, E. Monzani and F. Chillemi, J. Chem. Soc., Dalton Trans., 2945 (1991); https://doi.org/10.1039/dt9910002945.

10. Md. Mahbubul Alam, J. Bangladesh Acad. Sci., 35, 61 (2011).

11. S. Srivastava and A. Kalam, Synth. React. Inorg. Met.-Org. Chem., 34, 1529 (2004); https://doi.org/10.1081/SIM-200026581.

12. A. Kumar, S. Malviya and S. Srivastava, Asian J. Res. Chem, 9, 277 (2016); https://doi.org/10.5958/0974-4150.2016.00045.6.

13. A. Kumar, S. Malviya, R.K. Kushwaha, R. Kumar and S. Srivastava, Int. J. Chem. Phys. Sci., 5, 35 (2016).

14. H. Keypour, M. Rezaeivala, L. Valencia and P. Perez-Lourido, Polyhedron, 27, 3172 (2008); https://doi.org/10.1016/j.poly.2008.07.012.

15. H. Keypour, M. Rezaeivala, L. Valencia, S. Salehzadeh, P. Perez-Lourido and H.R. Khavasi, Polyhedron, 28, 3533 (2009); https://doi.org/10.1016/j.poly.2009.05.083.

16. S. Srivastava, Polyhedron, 4, 1925 (1985); https://doi.org/10.1016/S0277-5387(00)86712-1. 